\title{
A THEOREM OF THE ALTERNATIVE AND A TWO-FUNCTION MINIMAX THEOREM
}

\author{
ANTON STEFANESCU
}

Received 8 April 2003 and in revised form 19 January 2004

The two main results of the paper are a theorem of the alternative of Gordan type and a two-function minimax theorem. Both are based on some weakened convexlike properties, without any vector space structure.

\section{Introduction}

"Theorem of the alternative" is the generic name of different results used as an important tool in optimization. Though the early versions have been obtained under strong conditions of convexity, for the further extensions, these conditions have been weakened using generalized convexity beyond vector space structure. This is the case of the result proved by Jeyakumar [4], who used an extension of Fan's convexlike property.

Our theorem of the alternative, proved in Section 3, extends the cited result in the case of the usual order of the $n$-dimensional Euclidean space, and makes use of the weakened convexlike property introduced in Section 2.

The two-function minimax theorem extends the classical minimax inequality to the case of two functions. It is motivated both by the equilibrium problem in the noncooperative game theory and by the generalized duality in optimization. The first known result is due to Fan [3]. Several authors, especially in the last two decades, produced different versions of this theorem, employing the basic techniques from single-function minimax theory. We refer the reader to Simons [9] for a valuable survey in this framework. New results, based mainly on special connectedness properties, are obtained by Kindler in [5].

The main theorem of Section 4 is based on the theorem of the alternative proved in Section 3. For both results, one requires neither vector space structures nor topological support.

\section{Weakened convexlike properties}

Everywhere in this section, $\mathscr{F}$ will designate a family of real-valued functions defined on an arbitrary nonvoid set $X$. For any set $A$, we will denote by $\sigma(A)$ the family of all finite 
subsets of $A$, and, if $E$ is a subset of a linear space, then $\operatorname{co} E$ stands for the convex hull of $E$. Denote also by $\Delta_{n}$ the standard $(n-1)$-dimensional simplex.

We briefly review some notions which extend the classical convexity beyond vector spaces.

Let $t \in[0,1]$.

Definition 2.1. F्F is $t$-convexlike on $X$ if

$$
\forall x_{1}, x_{2} \in X, \exists x_{0} \in X, \forall f \in \mathscr{F}, \quad f\left(x_{0}\right) \leq t f\left(x_{1}\right)+(1-t) f\left(x_{2}\right) .
$$

Definition 2.2. $\mathscr{F}$ is $t$-subconvexlike on $X$ if

$$
\forall x_{1}, x_{2} \in X, \forall \epsilon>0, \exists x_{0} \in X, \forall f \in \mathscr{F}, \quad f\left(x_{0}\right) \leq t f\left(x_{1}\right)+(1-t) f\left(x_{2}\right)+\epsilon .
$$

Definition 2.3. $\mathscr{F}_{\mathscr{F}}$ is $t$-concavelike ( $t$-subconcavelike) on $X$ if $-\mathscr{F}$ is $t$-convexlike $(t$ subconvexlike) on $X$.

Definition 2.4. $\mathscr{F}$ is convexlike (subconvexlike) on $X$ if it is $t$-convexlike ( $t$-subconvexlike) on $X$ for all $t \in[0,1]$. $\mathscr{F}$ is concavelike (subconcavelike) on $X$ if $-\mathscr{F}$ is convexlike (subconvexlike) on $X$.

The concept of convexlike functions is due to Fan [3]. Weakening this property, König [6] introduced $t$-convexlike functions (for $t=1 / 2$ ) in order to extend Fan's minimax theorem. The concept of $t$-subconvexlike functions originates from Craven and Jeyakumar [2] but, in a particular form, it was introduced in Neumann [7].

Remark 2.5. If $\mathscr{F}$ is $t$-convexlike ( $t$-subconvexlike), then every subfamily $\mathscr{F}^{\prime}$ of $\mathscr{F}$ is also $t$-convexlike ( $t$-subconvexlike).

Remark 2.6. If $\mathscr{F}_{F}$ is $t$-convexlike ( $t$-subconvexlike) for some $t \in(0,1)$, then $\mathscr{F}_{F}$ is $t^{\prime}$ convexlike ( $t^{\prime}$-subconvexlike) for all $t^{\prime}$ in a set $D(t)$, dense in $[0,1]$. Obviously, $\{0,1\} \subset$ $D(t)$.

Remark 2.7. If $\mathscr{F}$ is $t$-convexlike ( $t$-subconvexlike), then co $\mathscr{F}$ is also $t$-convexlike $(t$ subconvexlike).

Remark 2.8. If $\mathscr{F}$ is $t$-convexlike for some $t \in(0,1)$, then for any integer $n \geq 2$ there exists $D_{n}(t)$, dense in $\Delta_{n}$, such that

$$
\begin{gathered}
\forall x_{1}, x_{2}, \ldots, x_{n} \in X, \forall\left(a_{1}, a_{2}, \ldots, a_{n}\right) \in D_{n}(t), \\
\exists x_{0} \in X, \forall f \in \mathscr{F}, \quad f\left(x_{0}\right) \leq \sum_{i=1}^{n} a_{i} f\left(x_{i}\right) .
\end{gathered}
$$

Similarly, if $\mathscr{F}$ is $t$-subconvexlike for some $t \in(0,1)$, then for any integer $n \geq 2$ there exists $D_{n}(t)$, dense in $\Delta_{n}$, such that

$$
\begin{gathered}
\forall x_{1}, x_{2}, \ldots, x_{n} \in X, \forall a=\left(a_{1}, a_{2}, \ldots, a_{n}\right) \in D_{n}(t), \forall \epsilon>0, \\
\exists x_{0} \in X, \forall f \in \mathscr{F}, \quad f\left(x_{0}\right) \leq \sum_{i=1}^{n} a_{i} f\left(x_{i}\right)+\epsilon .
\end{gathered}
$$


For other relationships between convexlike properties we refer to Paeck [8].

Based on the above remarks, we arrive to the following definitions (Stefanescu [11]).

Definition 2.9. F $\mathscr{F}_{\text {is }}$-weakly convexlike on $X$ if

$$
\forall x_{1}, x_{2} \in X, \quad \inf _{x \in X} \sup _{f \in \mathscr{F}} f(x) \leq \sup _{f \in \mathscr{F}}\left[t f\left(x_{1}\right)+(1-t) f\left(x_{2}\right)\right]
$$

$\mathscr{F}$ is weakly convexlike on $X$ if it is $t$-weakly convexlike for all $t \in[0,1]$.

Remark 2.10. $\mathscr{F}_{\text {is }}$-weakly convexlike for some $t$ if and only if co $\mathscr{F}$ is $t$-weakly convexlike.

Definition 2.11. $\mathscr{F}$ is affine weakly convexlike on $X$ if

$$
\forall x_{1}, x_{2}, \ldots, x_{n} \in X, \forall\left(t_{1}, t_{2}, \ldots, t_{n}\right) \in \Delta_{n}, \quad \inf _{x \in X} \sup _{f \in \mathscr{F}} f(x) \leq \sup _{f \in \mathscr{F}} \sum_{i=1}^{n} t_{i} f\left(x_{i}\right) .
$$

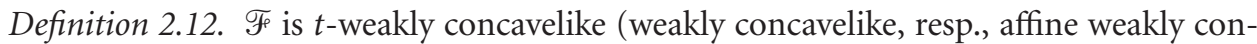
cavelike) if $-F$ is $t$-weakly convexlike (weakly convexlike, resp., affine weakly convexlike).

Remark 2.13. It follows by Remarks $2.6,2.7$, and 2.8 that if $\mathscr{F}$ is $t$-convexlike $(t$ subconvexlike) for some $t \in(0,1)$, then $\mathscr{F}$ and co $\mathscr{F}$ are weakly convexlike. Also, if $\mathscr{F}_{F}$ is convexlike (subconvexlike), it is affine weakly convexlike.

For the converse implications, consider the following simple counterexamples.

Example 2.14. Let $\mathbb{Z}^{*}$ be the set of all nonzero integers. Take $X=\left\{1 / z \mid z \in \mathbb{Z}^{*}\right\}$ and $\mathscr{F}=\left\{f_{a} \mid a \in \mathbb{Z}^{*}\right\}$, where $f_{a}(x)=x / a$.

One easily verifies that $\sup _{f \in \mathscr{F}} f(x)=\sup _{f \in \operatorname{co} \mathscr{F}} f(x)=|x|$ and then

$$
\inf _{x \in X} \sup _{f \in \mathscr{F}} f(x)=\inf _{x \in X} \sup _{f \in \operatorname{co} \mathscr{F}} f(x)=0 .
$$

On the other hand, for any $x_{1}, x_{2} \in X$ and $t \in[0,1], \sup _{f \in \mathscr{F}}\left[t f\left(x_{1}\right)+(1-t) f\left(x_{2}\right)\right]=$ $\sup _{f \in \operatorname{co} \mathscr{F}}\left[t f\left(x_{1}\right)+(1-t) f\left(x_{2}\right)\right]=\left|t x_{1}+(1-t) x_{2}\right|$. Thus, both $\mathscr{F}$ and co $\mathscr{F}$ are weakly convexlike.

Moreover, $\operatorname{since} \sup _{f \in \mathscr{F}} \sum_{i=1}^{n} t_{i} f\left(x_{i}\right)=\left|\sum_{i=1}^{n} t_{i} x_{i}\right|$ for every $\left(t_{1}, t_{2}, \ldots, t_{n}\right) \in \Delta_{n}$ and for any $x_{1}, x_{2}, \ldots, x_{n} \in X$, it follows that $\mathscr{F}$ is affine weakly convexlike.

To show that $\mathscr{F}$ is not $t$-subconvexlike, take $x_{1}=-1, x_{2}=1, t \in(0.05,0.1)$, and $\epsilon=$ 0.05. If $f_{a}(x) \leq\left[t f_{a}\left(x_{1}\right)+(1-t) f_{a}\left(x_{2}\right)\right]+\epsilon$ for all $a \in \mathbb{Z}^{*}$, then $x$ and $t$ must verify $2 t-1.05 \leq x \leq 2 t-0.95$. Obviously, for each $t$ in the considered interval, there are no $x \in X$ verifying this condition. Thus, by Remark 2.6 , there is no $t \in(0,1)$ such that $\mathscr{F}_{F}$ is $t$-subconvexlike ( $t$-convexlike). 
172 Alternative theorem and minimax inequality

Example 2.15. Let $\mathbb{N}^{*}$ be the set of all positive integers. Take $X=\left\{1 / n \mid n \in \mathbb{N}^{*}\right\}$ and $\mathscr{F}=\left\{f_{a} \mid a \in \mathbb{N}^{*}\right\}$, where

$$
f_{a}(x)= \begin{cases}a & \text { if } x<\frac{1}{a} \\ x & \text { if } x \geq \frac{1}{a}\end{cases}
$$

Since $\inf _{f \in \mathscr{F}} f(x)=\inf _{f \in \operatorname{co} \mathscr{F}} f(x)=x$, then

$$
\sup _{x \in X} \inf _{f \in \mathscr{F}} f(x)=\sup _{x \in X} \inf _{f \in \operatorname{co} \mathscr{F}} f(x)=1 .
$$

Also, $\inf _{f \in \operatorname{co} \mathscr{F}} \sum_{i=1}^{n} t_{i} f\left(x_{i}\right)=\sum_{i=1}^{n} t_{i} x_{i}$ for every $t=\left(t_{1}, t_{2}, \ldots, t_{n}\right) \in \Delta_{n}$ and for any $x_{1}$, $x_{2}, \ldots, x_{n} \in X$.

Hence, co $\mathscr{F}(\mathscr{F})$ is weakly concavelike and $\mathscr{F}$ is affine weakly concavelike.

However, $\mathscr{F}$ is not $t$-subconcavelike for any $t \in(0,1)$. One can verify that there does not exist a dense set $D \subseteq[0,1]$ such that, for every $t \in D$, the inequality

$$
f_{a}(x) \geq\left[t f_{a}(1)+(1-t) f_{a}\left(\frac{1}{3}\right)\right]-\epsilon
$$

admits solutions in $X$ independent of $a$, if $\epsilon$ is small enough.

For instance, if $\epsilon<1 / 12$, there are no $t$ in the interval $(3 \epsilon / 2,1-\epsilon)$ satisfying (2.10) for an appropriate $x \in X$ and all $a \in \mathbb{N}^{*}$.

\section{A general theorem of the alternative}

Theorem 3.1. Let $\mathscr{F}$ be a finite family of real-valued functions defined on a nonvoid set $X$. If every $\mathscr{H} \in \sigma(\operatorname{co} \mathscr{F})$ is weakly convexlike, then exactly one of the following two situations occurs:

$$
\begin{array}{cc}
\exists \bar{x} \in X, \forall f \in \mathscr{F}, & f(\bar{x})<0, \\
\exists \bar{f} \in \operatorname{co} \mathscr{F}, \forall x \in X, & \bar{f}(x) \geq 0 .
\end{array}
$$

The proof of the theorem is based on the following three lemmas.

Lemma 3.2. Let $f_{1}, f_{2}$ be any real-valued functions on $X$ satisfying the following two conditions:

$$
\begin{gathered}
\forall x \in X, \quad \max _{i=1,2} f_{i}(x) \geq 0, \\
\forall t \in[0,1], \quad \varnothing \in\{\varphi(t) \cap \varphi(0), \varphi(t) \cap \varphi(1)\},
\end{gathered}
$$

where $\varphi(t)=\left\{x \in X \mid t f_{1}(x)+(1-t) f_{2}(x)<0\right\}, t \in[0,1]$.

Then, there exists $t_{0} \in[0,1]$ such that

$$
\forall x \in X, \quad t_{0} f_{1}(x)+\left(1-t_{0}\right) f_{2}(x) \geq 0 .
$$


Proof. Obviously, $\varphi(t) \subseteq \varphi(0) \cup \varphi(1)$ for every $t \in[0,1]$. Denote $\varphi^{-1}(A)=\{t \in[0,1] \mid$ $\varphi(t) \cap A \neq \varnothing\}, A \subseteq X$. By (3.2) and (3.3) it results that $\varphi^{-1}(\varphi(0)) \cap \varphi^{-1}(\varphi(1))=\varnothing$. Obviously, $0 \in \varphi^{-1}(\varphi(1)), 1 \in \varphi^{-1}(\varphi(0))$ and these two sets are open in [0,1]. It follows that $[0,1] \backslash\left(\varphi^{-1}(\varphi(0)) \cup \varphi^{-1}(\varphi(1))\right) \neq \varnothing$ so that there exists $t_{0} \in[0,1]$ with $\varphi\left(t_{0}\right)=\varnothing$.

Lemma 3.3. Let $f_{1}, f_{2}$ satisfy (3.2). If F $=\left\{f_{1}, f_{2}\right\}$ is weakly convexlike, then (3.3) holds.

Proof. By way of contradiction, we assume that there exists $t \in[0,1], x_{1}, x_{2} \in X$, such that $f_{1}\left(x_{1}\right)<0, f_{2}\left(x_{2}\right)<0$ and

$$
\begin{aligned}
& t f_{1}\left(x_{1}\right)+(1-t) f_{2}\left(x_{1}\right)<0 \\
& t f_{1}\left(x_{2}\right)+(1-t) f_{2}\left(x_{2}\right)<0
\end{aligned}
$$

Denote $a_{i}=f_{1}\left(x_{i}\right), b_{i}=f_{2}\left(x_{i}\right), i=1,2$. Obviously, (3.5) imply that

$$
\frac{b_{1}}{b_{1}-a_{1}}<\frac{b_{2}}{b_{2}-a_{2}}
$$

which is equivalent to

$$
\frac{a_{2}}{a_{2}-a_{1}}<\frac{b_{2}}{b_{2}-b_{1}}
$$

Then, for every $t^{\prime} \in\left(a_{2} /\left(a_{2}-a_{1}\right), b_{2} /\left(b_{2}-b_{1}\right)\right)$, it results that

$$
\begin{aligned}
& t^{\prime} f_{1}\left(x_{1}\right)+\left(1-t^{\prime}\right) f_{1}\left(x_{2}\right)<0, \\
& t^{\prime} f_{2}\left(x_{1}\right)+\left(1-t^{\prime}\right) f_{2}\left(x_{2}\right)<0 .
\end{aligned}
$$

Now, since $\left\{f_{1}, f_{2}\right\}$ is $t^{\prime}$-weakly convexlike, the last two inequalities imply that $\inf _{x \in X} \max _{i=1,2} f_{i}(x)<0$. Thus, there exists $x_{0} \in X$ such that $\max _{i=1,2} f_{1}\left(x_{0}\right)<0$, contradicting (3.2).

Lemma 3.4. Let $\mathscr{F}=\left\{f_{1}, f_{2}, \ldots, f_{n}\right\}, n \geq 3$, be weakly convexlike on $X$. Then $f_{1}$ and $f_{2}$ satisfy (3.3) with respect to the set

$$
X^{\prime}=\left\{x \in X \mid f_{i}(x)<0, i=3, \ldots, n\right\},
$$

whenever $\max _{i=1,2} f_{i}(x) \geq 0$, for all $x \in X^{\prime}$.

Proof. As in the proof of Lemma 3.3, choose $t^{\prime}$ and observe that

$$
\forall i=1,2, \ldots, n, \quad t^{\prime} f_{i}\left(x_{1}\right)+\left(1-t^{\prime}\right) f_{i}\left(x_{2}\right)<0
$$

Note that condition (3.3) was first used in Stefanescu [10] for proving a general minimax theorem. 
Proof of Theorem 3.1. The proof follows by induction on $n=|\mathscr{F}|$.

For $n=2$, the alternative (3.1) follows directly from Lemmas 3.2 and 3.3.

Assume that the theorem holds for every family of at most $n-1$ functions, $n \geq 3$, and let $\mathscr{F}_{F}=\left\{f_{1}, f_{2}, \ldots, f_{n}\right\}$ satisfy $\max _{1 \leq i \leq n} f_{i}(x) \geq 0$ for all $x \in X$.

Denote $X^{\prime}=\left\{x \in X \mid f_{i}(x)<0, i=3, \ldots, n\right\}$. If $X^{\prime}=\varnothing$, then the conclusion follows from the inductive assumption. Otherwise, $\max _{i=1,2} f_{i}(x) \geq 0$ for all $x \in X^{\prime}$. Then, by Lemma 3.4, it follows that $\left\{f_{1}, f_{2}\right\}$ satisfies the assumptions of Lemma 3.2 with respect to $X^{\prime}$. Hence, there exists $f_{0} \in \operatorname{co}\left\{f_{1}, f_{2}\right\}$ such that $f_{0}(x) \geq 0$ for all $x \in X^{\prime}$. Moreover, $\max \left\{f_{0}(x), f_{3}, \ldots, f_{n}(x)\right\} \geq 0$ for all $x \in X$. By induction, there exists $\bar{f} \in \operatorname{co}\left\{f_{0}, f_{3}, \ldots\right.$, $\left.f_{n}\right\}=\operatorname{co} \mathscr{F}$ with $\bar{f}(x) \geq 0$, for all $x \in X$.

By Remark 2.10, co $\mathscr{F}$ is weakly convexlike if $\mathscr{F}$ is weakly convexlike. But unlike in the case of previously known convexlike properties, a subfamily of a weakly convexlike family of functions is not necessarily weakly convexlike too so that in Theorem 3.1 it is not sufficient to assume that $\mathscr{F}$ is weakly convexlike. However, we can observe that all that we need for proving Theorem 3.1 is the following condition.

(C) There exists an ordering $\left\{f_{1}, f_{2}, \ldots, f_{n}\right\}$ of $\mathscr{F}$, such that the family of functions $\left\{\bar{f}, f_{k+1}, \ldots, f_{n}\right\}$ is weakly convexlike, for every $k=1, \ldots, n-1$ and $\bar{f} \in \operatorname{co}\left(\left\{f_{1}, \ldots, f_{k}\right\}\right)$.

The following corollary of Theorem 3.1 easily follows from Remarks 2.13 and 2.5. It is not else than the alternative theorem of Jeyakumar [4] for the usual (partial) order of $\mathbb{R}^{n}$.

Corollary 3.5. Let $\mathscr{F}$ be $t$-subconvexlike for some $t \in(0,1)$. Then the alternative (3.1) holds.

Example 2.14 can be used once more to show that Theorem 3.1 generalizes previously known alternative theorems concerning convexlike-type functions.

It is easy to see that the alternative (3.1) holds for any finite subset of $\mathscr{F}$ (and of co $\mathscr{F}$ as well), but the conditions of Corollary 3.5 are not satisfied. Obviously, co $\mathscr{F}_{\mathcal{F}}=\left\{f_{\alpha} \mid \alpha \in\right.$ $[-1,1]\}$, where $f_{\alpha}(x)=\alpha x$. Arguing as in Example 2.14, one can verify that any finite subfamily $\mathscr{F}^{\prime}=\left\{f_{\alpha_{i}} \mid 1 \leq i \leq k\right\}$, where $-1 \leq \alpha_{1}<\cdots<\alpha_{k} \leq 1$ and $\alpha_{1}<0<\alpha_{k}$, is not $t$-subconvexlike for any $t \in(0,1)$, so that Corollary 3.5 fails.

On the other hand, we can show that Theorem 3.1 can be applied in this example. All that we must do is to verify that any $\mathscr{F}^{\prime}=\left\{f_{\alpha_{i}} \mid 1 \leq i \leq k\right\}$ is affine weakly convexlike.

Fix $n, t \in \Delta_{n}$ and $x^{1}, \ldots, x^{n} \in X$. Set $\bar{x}=\sum_{j=1}^{n} t_{j} f_{\alpha_{i}}\left(x^{j}\right)$. The inequality

$$
\inf _{x \in X} \max _{1 \leq i \leq k} f_{\alpha_{i}}(x) \leq \max _{1 \leq i \leq k} \sum_{j=1}^{n} t_{j} f_{\alpha_{i}}\left(x^{j}\right)
$$

is equivalent to the inequality $\inf _{x \in X} \max _{1 \leq i \leq k} \alpha_{i} x \leq \max _{1 \leq i \leq k} \alpha_{i} \bar{x}$ and the latter results from a simple calculation.

Remark 3.6. We may ask whether Jeyakumar's alternative theorem can be generalized in the more abstract framework of an order induced by an arbitrary convex cone. The appropriate reformulation of Definition 2.9 in terms of such order is an open problem. Moreover, since our approach involves only quantitative relations, the technique of proof is inadequate in an abstract framework. 


\section{A two-function minimax theorem}

The main result of this section is obtained via the previous theorem of the alternative.

Theorem 4.1. Let $X, Y$ be any nonvoid sets and $f, g$ two real-valued functions defined on the product set $X \times Y$, such that

$$
\forall x_{1}, \ldots, x_{n} \in X, \forall\left(t_{1}, \ldots, t_{n}\right) \in \Delta_{n}, \quad \sup _{x \in X} \inf _{y \in Y} g(x, y) \geq \inf _{y \in Y} \sum_{i=1}^{n} t_{i} f\left(x_{i}, y\right) .
$$

If

$$
\text { each } \mathscr{H} \in \sigma(\operatorname{co} \mathscr{F}) \text { is weakly convexlike on } Y \text {, }
$$

where $\mathscr{F}=(f(x, \cdot))_{x \in X}$, then

$$
\sup _{X^{\prime} \in \sigma(X)} \inf _{y \in Y} \max _{x \in X^{\prime}} f(x, y) \leq \sup _{x \in X} \inf _{y \in Y} g(x, y) .
$$

Proof. Let $X^{\prime} \in \sigma(X)$ and suppose that $\inf _{y \in Y} \max _{x \in X^{\prime}} f(x, y) \geq \alpha$, for some $\alpha$. Apply the theorem of the alternative to $(f(x, \cdot)-\alpha)_{x \in X^{\prime}}$. It follows the existence of the nonnegative numbers $t_{x}, x \in X^{\prime}, \sum_{x \in X^{\prime}} t_{x}=1$, such that

$$
\sum_{x \in X^{\prime}} t_{x} f(x, y) \geq \alpha, \quad \forall y \in Y
$$

But then, by (4.1) it follows that

$$
\sup _{x \in X} \inf _{y \in Y} g(x, y) \geq \alpha
$$

Therefore,

$$
\sup _{x \in X} \inf _{y \in Y} g(x, y) \geq \inf _{y \in Y} \max _{x \in X^{\prime}} f(x, y) \text {. }
$$

Since $X^{\prime}$ is any finite subset of $X$, this proves the theorem.

For the case when $f(x, y) \leq g(x, y)$, for all $(x, y) \in X \times Y$, which is usually considered in the two-function minimax theorems, we easily obtain the following theorem.

Theorem 4.2. Let $X, Y$ be any nonvoid sets and $f, g$ two real-valued functions defined on the product set $X \times Y$ such that $f(x, y) \leq g(x, y)$ for all $(x, y) \in X \times Y$. If (4.2) holds, and

$\varphi$ is affine weakly concavelike on $X$,

where $\varphi=(g(\cdot, y))_{y \in Y}$, then (4.3) holds.

Now, the two-function minimax inequality

$$
\inf _{y \in Y} \sup _{x \in X} f(x, y) \leq \sup _{x \in X} \inf _{y \in Y} g(x, y)
$$


can be obtained if one combines the assumptions of the previous theorems with any sufficient conditions for the inequality

$$
\inf _{y \in Y} \sup _{x \in X} f(x, y) \leq \sup _{X^{\prime} \in \sigma(X)} \inf _{y \in Y} \max _{x \in X^{\prime}} f(x, y)
$$

We denote $Y_{\alpha}(x)=\{y \in Y \mid f(x, y) \leq \alpha\}, x \in X, \alpha \in \mathbb{R}$.

Proposition 4.3. Assume that the following condition holds.

(*) There exists $\bar{\alpha} \geq \inf _{y \in Y} \sup _{x \in X} f(x, y)$ such that for all $\alpha \leq \bar{\alpha}$, the family $\left(Y_{\alpha}(x)\right)_{x \in X}$ has nonempty intersection whenever it has the finite intersection property.

Then (4.9) holds.

Proof. By way of contradiction, we assume that

$$
\inf _{y \in Y} \sup _{x \in X} f(x, y)>\sup _{X^{\prime} \in \sigma(X)} \inf _{y \in Y} \max f(x, y) .
$$

Pick an $\alpha \leq \bar{\alpha}$, inf $\inf _{y \in Y} \sup _{x \in X} f(x, y)>\alpha>\sup _{X^{\prime} \in \sigma(X)} \inf _{y \in Y} \max _{x \in X^{\prime}} f(x, y)$. This implies $\cap_{x \in X} Y_{\alpha}(x)=\varnothing$. From the finite intersection property, it follows that there exists $X^{\prime} \in$ $\sigma(X)$ such that $\cap_{x \in X^{\prime}} Y_{\alpha}(x)=\varnothing$. Thus, $\inf _{y \in Y} \max _{x \in X^{\prime}} f(x, y)>\alpha$.

Remark 4.4. Condition $(*)$ is fulfilled if $\left(Y_{\alpha}(x)\right)_{x \in X}$ are closed and compact in a topological space, for $\alpha \leq \bar{\alpha}$.

The proposition also holds if one assumes $Y_{\bar{\alpha}}(\bar{x})$ to be compact, for some $\bar{x} \in X$, and $Y_{\alpha}(x)$ closed in $X$, for all $x \in X$ and $\alpha \leq \bar{\alpha}$.

From Proposition 4.3 and Theorems 4.1 and 4.2, we easily obtain the following twofunction minimax theorems.

Theorem 4.5. Let $X, Y$ be any nonvoid sets and $f, g$ two real-valued functions defined on $X \times Y$. Assume condition ( $*$ ) to be satisfied. If (4.1) and (4.2) hold, then (4.8) holds.

Theorem 4.6. Let $X, Y$ be any nonvoid sets and $f, g$ two real-valued functions defined on the product set $X \times Y$, such that $f(x, y) \leq g(x, y)$ for all $(x, y) \in X \times Y$. Assume condition (*) to be satisfied. If (4.2) and (4.7) hold, then (4.8) holds.

Based on the examples of Section 2, one can see that the above results are independent of most known similar results.

An easy consequence of the last two theorems is a slight generalization of the twofunction minimax theorem proved by Cheng and Lin in [1].

Corollary 4.7. Let $X, Y$ be any nonvoid sets and $f, g$ two real-valued functions defined on the product set $X \times Y$, such that $f(x, y) \leq g(x, y)$ for all $(x, y) \in X \times Y$. Assume that there exists $t, s \in(0,1)$ such that $\mathscr{F}$ is $t$-subconvexlike and $\varphi$ is $s$-subconcavelike. If condition (*) holds, then (4.8) holds. 


\section{References}

[1] C.-Z. Cheng and B.-L. Lin, Nonlinear, noncompact minimax theorems, Bull. Inst. Math. Acad. Sinica 24 (1996), no. 1, 23-32.

[2] B. D. Craven and V. Jeyakumar, Alternative and duality theorems with weakened convexity, Utilitas Math. 31 (1987), 149-159.

[3] K. Fan, Sur un théorème minimax, C. R. Math. Acad. Sci. Paris 259 (1964), 3925-3928 (French).

[4] V. Jeyakumar, A generalization of a minimax theorem of Fan via a theorem of the alternative, J. Optim. Theory Appl. 48 (1986), no. 3, 525-533.

[5] J. Kindler, Two-function topological minimax theorems, J. Math. Anal. Appl. 225 (1998), no. 1, 312-325.

[6] H. König, Über das von neumannsche minimax-theorem, Arch. Math. (Basel) 19 (1968), 482487 (German).

[7] M. Neumann, Bemerkungen zum von neumannschen minimaxtheorem, Arch. Math. (Basel) 29 (1977), no. 1, 96-105 (German).

[8] S. Paeck, Convexlike and concavelike conditions in alternative, minimax, and minimization theorems, J. Optim. Theory Appl. 74 (1992), no. 2, 317-332.

[9] S. Simons, Minimax theorems and their proofs, Minimax and Applications (D.-Z. Du and P. M. Pardalos, eds.), Nonconvex Optim. Appl., vol. 4, Kluwer Academic Publishers, Dordrecht, 1995, pp. 1-23.

[10] A. Stefanescu, A general min-max theorem, Optimization 16 (1985), no. 4, 497-504.

[11] - Alternative and minimax theorems beyond vector spaces, J. Math. Anal. Appl. 264 (2001), no. 2, 450-464.

Anton Stefanescu: Faculty of Mathematics, Bucharest University, 14 Academiei Street, Bucharest 70109, Romania

E-mail address: anton@pro.math.unibuc.ro 


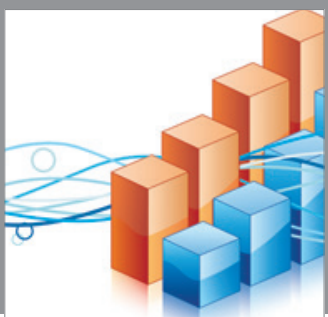

Advances in

Operations Research

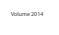

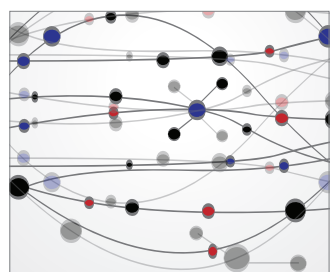

\section{The Scientific} World Journal
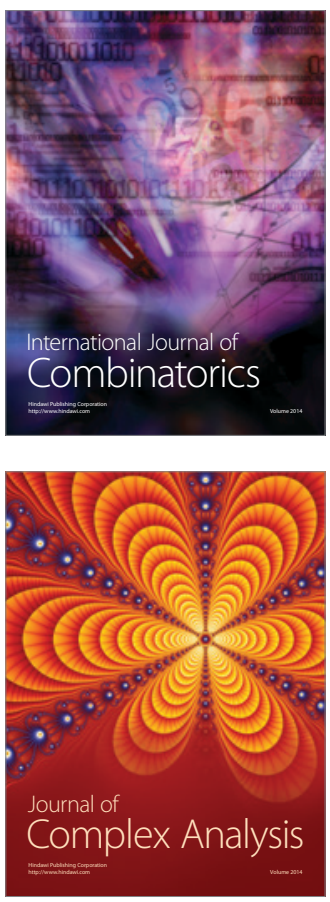

International Journal of

Mathematics and

Mathematical

Sciences
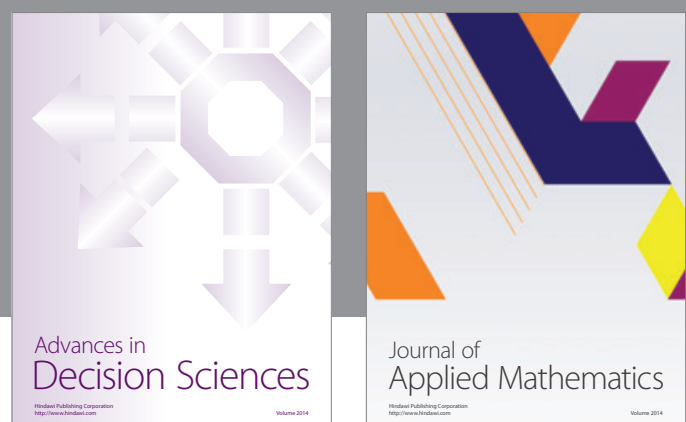

Journal of

Applied Mathematics
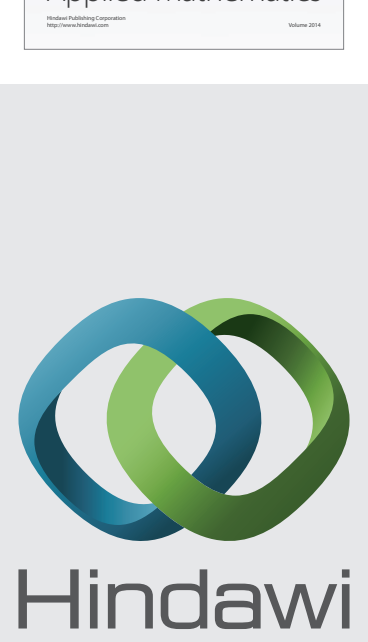

Submit your manuscripts at http://www.hindawi.com
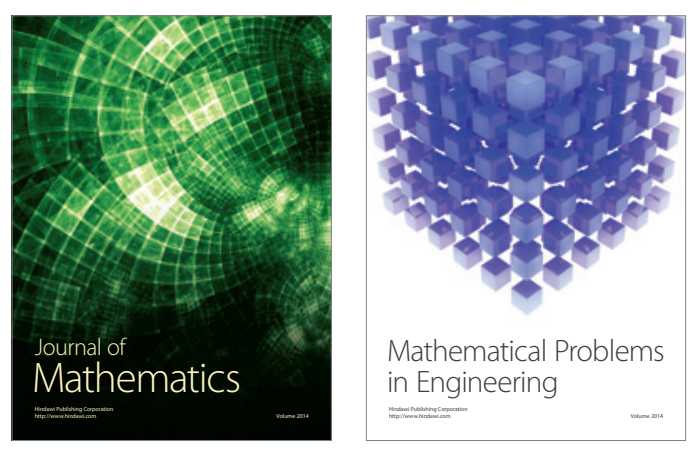

Mathematical Problems in Engineering
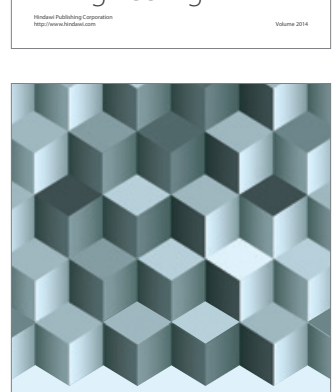

Journal of

Function Spaces
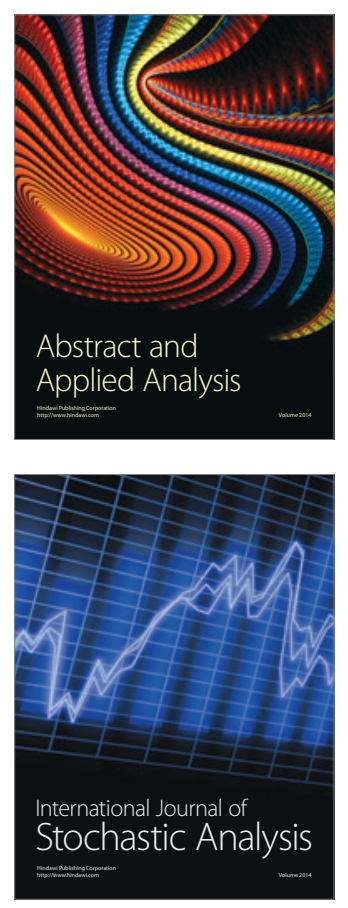

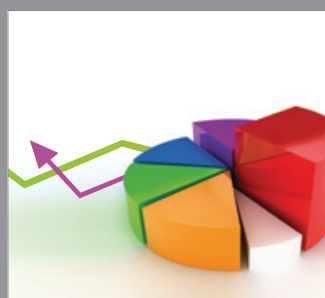

ournal of

Probability and Statistics

Promensencen
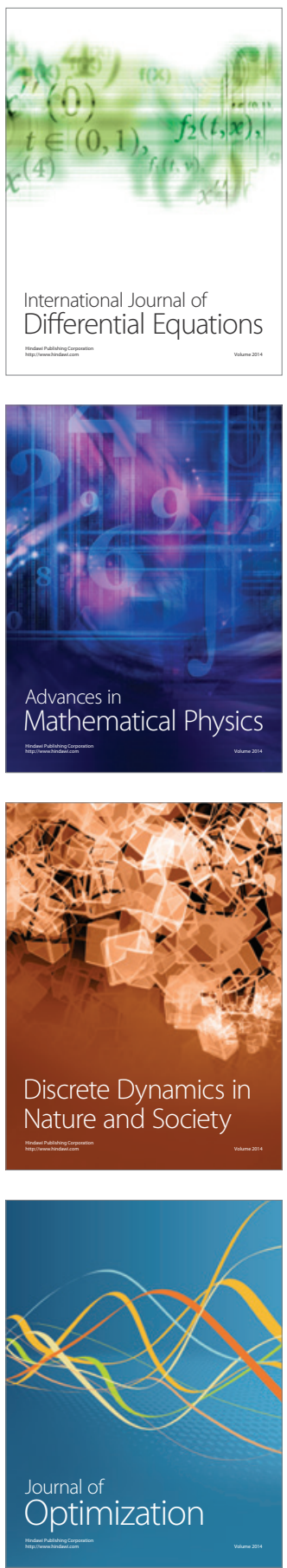